\title{
MALESTAR SOCIAL Y MALESTAR DOCENTE: UNA INVESTIGACIÓN SOBRE EL SÍNDROME DE DESGASTE PROFESIONAL BURNOUT Y SU INCIDENCIA SOCIOEDUCATIVA
}

\section{Social discomfort and teacher distress: a research of burnout syndrome and its socioeducative incidence}

\author{
David Sánchez Llull, Martí X. March y Cerdà y Lluís Ballester Brage \\ Departamento de Ciencias de la Educación. Universitat de les Illes Balears (UIB) \\ Correo-e:dsanchez@iesemilidarder.com; marti.march@uib.es; lluis.ballester@uib.es \\ Recepción: 13 de marzo de 2014 \\ Envío a informantes: 28 de mayo de 2014 \\ Fecha de aceptación definitiva: I9 de enero de 20I5 \\ Biblid. [0214-3402 (2015) (II época) n. ${ }^{\circ} 21 ; 245-257$ ]
}

REsumen: La comunidad escolar y los conflictos que en ella se generan están siendo objeto de preocupación, tanto por la sociedad como por la misma comunidad educativa. Los docentes tienen que dar respuesta a una sociedad cada vez más exigente con la educación, caracterizada por múltiples factores no solo educativos, sino también sociales. Este amplio abanico de variables a los que se enfrenta el docente está provocando cierto malestar por la aparición de múltiples problemas psicosociales como el síndrome de desgaste profesional o burnout. Presentamos una investigación que intenta determinar la prevalencia de esta problemática psicosocial en los centros públicos de secundaria de las Illes Balears, a partir del estudio de tres variables: el cansancio emocional, la despersonalización y la realización personal, utilizando como instrumento el MBI de Maslach y Jackson.

Palabras clave: síndrome de desgaste profesional; malestar social; malestar docente; burnout; variables; evaluación; Illes Balears.

AвstRAct: The school community and the conflicts that are generated in there, are being the subject of concern, both by society as by the same educational community. Teachers have to respond to a society an increasingly demanding with education, characterized by multiple factors not only educational but also social. This wide range of variables to which the teacher faces is causing some discomfort by the appearance of multiple psychosocial problems such as burnout syndrome. We present research that attempts to determine the prevalence of this psychosocial problematic in public secondary schools in the Balearic Islands, from the study of three variables: emotional 
exhaustion, depersonalization and personal accomplishment, using as instrument the MBI Maslach and Jackson.

KEY WORDS: social malaise; educational malaise; burnout; variables; evaluation; Balearic Islands.

\section{Introducción: el malestar social y el malestar docente}

$\mathrm{L}$

A CONVIVENCIA ESCOLAR Y LOS CONFLICTOS ESCOLARES están siendo objeto, cada vez más, de preocupación por parte de la sociedad y de la misma comunidad educativa. Efectivamente, existen diversas razones para hablar de convivencia escolar en estos momentos, debido a que los centros de enseñanza tienen que ser lugares seguros para el aprendizaje; para evitar la vulneración de derechos básicos como son los derechos de menores, del profesorado y de las familias, el derecho a la educación, a la igualdad de oportunidades, al respeto y a la dignidad, entre otros. En esta situación de crisis económica, la preocupación por la educación en sus diversas manifestaciones se está acrecentando de forma significativa, a nivel político, a nivel social y a nivel de comunidad educativa. La cuestión escolar, en esta época de recortes educativos y de cuestionamiento real del Estado y de la sociedad del bienestar, es cada vez más objeto de debate y de polémica quedando reflejado en el informe de la ocDE (Ministerio de Educación, Cultura y Deportes, 20I2) el cual manifiesta que España necesita mejorar más la Educación Secundaria: «La segunda etapa de educación secundaria... se debe mejorar más, pues no es suficiente para cerrar el déficit de titulados en España en estudios secundarios».

Por otro lado, los docentes tienen que dar respuesta a una sociedad que quiere una escuela cada vez más exigente y de mejor calidad, donde se educa a través de diferentes idiomas y de estudiantes de diversas procedencias, donde los docentes tienen en cuenta cuestiones culturales y de género, promueven la tolerancia y la cohesión social, dan respuesta con eficacia a los alumnos desfavorecidos o con problemas de aprendizaje y de conducta, utilizan las nuevas tecnologías; se deben mantener actualizados en sus diferentes áreas de conocimiento y en las formas de evaluar... factores que, no lo debemos olvidar, pueden tener también efectos de forma directa (bien en positivo o bien en negativo) sobre sus propias motivaciones (OCDE, 2009).

Desde esta perspectiva de la problemática actual de educación, una de las cuestiones que cada vez más se está poniendo de manifiesto, a través de análisis y estudios basados sobre la evidencia, es la existencia de un creciente malestar entre los docentes, como consecuencia de muchos factores, los cuales tienen cada vez más repercusión social al ser expuestos a la comunidad educativa como, por ejemplo, las aportaciones del sindicato ANPE, el cual manifiesta en la Memoria Estatal del Defensor del Profesor (Asociación Nacional de Profesionales de la Enseñanza, 20I3) que, después de 8 años de funcionamiento del teléfono del defensor del profesorado, este proyecto ha atendido 22.313 incidencias, donde el 50\% de éstas hacían referencia a la ansiedad, un 16\% manifestaba depresión y un 10\% estaban relacionadas con solicitudes de baja laboral. Por otra parte, el tema se ha consolidado con fuerza en 
las Illes Balears a partir de la denuncia de cc. Oo.' (20I4) sobre las problemáticas psicosociales que afectan a los docentes.

A partir de aquí, es importante manifestar que no queremos en este artículo hacer un estudio exhaustivo sobre dicho malestar docente, pero sí analizar una de las expresiones del mismo: el síndrome de desgaste profesional o burnout. Efectivamente, cuando se habla de malestar docente se hace referencia a un fenómeno que afecta a una buena parte del profesorado y que se origina en respuesta a una serie de factores o acontecimientos negativos que aquejan a dicho profesorado en el marco educativo. Tal respuesta vendría precedida de un estilo atributivo pesimista que sería el responsable del incremento de los índices de estrés laboral burnout y ansiedad que exhiben los docentes afectados. Por tanto, la relación entre el malestar docente y el burnout es algo que la literatura científica sobre el tema ha puesto de manifiesto de forma clara y evidente (Esteve, 1987; Prieto y Bermejo, 2005; Organización Internacional del Trabajo, 20II).

Con todo, esa relación entre el malestar docente y el burnout también implica analizar las relaciones entre esta problemática y el malestar social, los problemas sociales de la educación; así, en la escuela no sólo se ponen de manifiesto los diferentes problemas sociales, sino que los condicionantes y los productos de la educación sólo se pueden entender desde este análisis. Los estudios tanto de la sociología de la educación como de la psicología social de la educación están incidiendo sobre la dimensión social de la educación, a través del análisis de las diversas variables.

Pero, además, este estudio -sobre todo en sus consecuencias, pero también en sus causas y manifestaciones- pretende incidir sobre los aspectos socioeducativos de dicha problemática, tanto en relación a su explicación, como a su solución. En este sentido, contextualizamos este estudio en el marco de la Pedagogía Social, más en concreto, de lo que denominamos Pedagogía Social Escolar. Efectivamente, aunque la Pedagogía Social se ha desarrollado en el ámbito de la educación no escolar, existe una corriente que plantea, dentro de la redefinición de la Pedagogía Escolar, la integración de la escuela por sus funciones, por sus manifestaciones, y por el carácter socializador de la escuela en todos los sentidos (Ortega, 2002; Parcerisas, 2008).

La cuestión del burnout, también, puede ser analizada desde la perspectiva de la Pedagogía Social, al menos, desde la perspectiva no sólo de su incidencia socioeducativa, sino de su resolución socioeducativa.

\section{La realidad del burnout}

El síndrome de desgaste profesional burnout, lejos de ser un término moderno, aparece mencionado ya en el año 1960 (Greene, 1960), en una novela titulada $A$ burn out case, donde se describe el agotamiento emocional y las consecuencias que se derivan de ella en un trabajador que pierde la fe en lo que hace. Pasados unos años, Freudenberg, en 1974, nos introduce en el concepto a partir de su estudio sobre el estrés e intenta conceptualizar el burnout desde el punto de vista clínico para diferenciarlo de otras enfermedades. A partir de aquí, la Asociación Americana de Psicología mediante

Ver http://feccoo-ib.org/comunes/recursos/I5684/I759307-Denuncia_contra_la_Conselleria_ presen-tada_a_registre_inspeccio_de_treball.pdf. 
Maslach (1976) lo describe desde una perspectiva psicológica y es esta autora, junto a Jackson (I98I), la que lo define como estrés laboral crónico, relacionando este nuevo concepto con tres dimensiones que determinan su aparición: cansancio emocional, despersonalización y reducido logro personal, que puede ocurrir en individuos que de alguna manera trabajan con personas (Maslach y Jackson, 1982). Ya en los años 90, Gil-Monte y Peiró desde una perspectiva psicosocial manifiestan que el burnout aparece cuando fallan las estrategias de afrontamiento de los problemas a nivel personal y organizacional (Gil-Monte y Peiró, 1996). Es en esta década cuando empieza a relacionarse el burnout con la prevención de los riesgos laborales, seguridad y salud, apareciendo en España la Ley 3I/1995, de 8 de noviembre, de Prevención de Riesgos Laborales, la cual inicia la consideración genérica del tema. Por otra parte, Farber en el año 2000 relaciona el burnout con la competencia laboral (Farber, 2000) y la OIT, mediante el Instituto Internacional de Estudios Internacionales, manifiesta que el burnout se ha de tener en cuenta para elaborar los planes de prevención de riesgos laborales, ya que estos inciden en la salud de los trabajadores y en consecuencia en sus resultados laborales (OIT, 20II).

Desde el año 2002, la Agencia Europea para la Seguridad y la Salud en el Trabajo (OsHA) ha puesto de manifiesto que casi uno de cada cuatro trabajadores europeos $(28 \%)$ sufre estrés en el trabajo. La citada agencia menciona que en la Unión Europea hay $4 \mathrm{I}$ millones de trabajadores que sufren estrés y que entre un 50 o $60 \%$ de las bajas laborales están relacionadas con esta causa (Agencia Europea para la Seguridad y Salud en el Trabajo, 2002: 6-8). Por tanto, el impacto social y económico del estrés y su derivación en estrés crónico laboral burnout es enorme. Además, si a esto le sumamos que las empresas, actualmente, manifiestan que el sistema educativo actual no forma bien a sus alumnos (Mourshed, Patel y Suder, 20I4), tenemos un caldo de cultivo propicio para el desastre, apareciendo indicadores como el desarrollado por el Sistema Estatal de Indicadores de la Educación de 2oro, que manifiesta que la esperanza de vida escolar media de un niño de seis años en las Illes Balears es la más baja de toda España, con una media de I2,I años, mientras que la media estatal es de I4,4 años. El mismo informe saca a la luz diferentes problemáticas como por ejemplo que las Illes Balears están a la cola en la competencia de comunicación lingüística, y también están por debajo de la media en competencia matemática y son de los últimos en competencia de conocimiento del medio físico. Por último, tenemos el número más bajo en graduados de Eso, bachiller y estudios superiores (Instituto de Evaluación, 20Io).

No es de extrañar que, a medida que el problema del burnout en los institutos de les Illes Balears va cobrando importancia debido a que afecta cada vez más al colectivo de profesionales encargado de la educación de nuestros jóvenes, aparezcan nuevas voces que intentan conocer, cuantificar y solventar este grave problema (Tortella-Feliu, 200I; Manasero, Vázquez, Ferrer, Fornés y Fernández, 2003), ya que el resultado de no hacer nada puede ser la incapacidad de desarrollar un proyecto educativo coherente, saludable y viable. El objetivo final de esta investigación es conocer las bases por las que se desarrolla el burnout, determinar en qué medida incide el síndrome del burnout en los profesores de centros públicos de las Illes Balears en la actualidad y si las medidas de prevención que se deben asumir serán adecuadas para poder desarrollar un modelo de escuela los más saludable posible.

De esta manera, el primer paso para empezar el estudio sobre el burnout ha sido analizar los informes que han realizado diferentes organismos internacionales sobre la 
problemática que nos ocupa. Tanto la Organización Mundial de la Salud (oms), la Red Mundial de Salud Ocupacional (GOHNET), la Organización Internacional del Trabajo (ОIт) y la Agencia Europea para la Seguridad y la Salud en el Trabajo (EU-OSHA) tienen como objetivo detectar nuevos riesgos en materia de salud y seguridad en el trabajo con la finalidad de aumentar su eficacia y el desarrollo de medidas preventivas. En lo referente a la definición que hacen estos organismos sobre el burnout, la osha (Agencia Europea para la Seguridad y Salud en el Trabajo, 20II) lo define como un síndrome caracterizado por el cansancio emocional, la alienación y una reducida capacidad laboral. Muy parecida es la definición que realiza la GOHNET, la cual menciona que «el síndrome de desgaste abarca las tres dimensiones siguientes: extenuación emocional; despersonalización y falta de realización personal» (Red Mundial de Salud Ocupacional, 200r: 5).

Es importante manifestar que no existe una única definición que sea compartida y aceptada por toda la comunidad científica sobre el burnout, sin embargo, la mayoría de investigadores y organismos internacionales aceptan de alguna manera la definición tripartita desarrollada por Maslach y Jackson que se enmarca dentro de la perspectiva psicosocial. Estas autoras definen el burnout como «un síndrome de agotamiento emocional, despersonalización y baja realización personal, que se puede dar entre individuos que trabajan con personas» (Maslach y Jackson, I986). Por lo tanto, encontramos tres factores que utilizaremos como variables de estudio, que inciden en este síndrome y que quedan definidos de la siguiente manera (Longás, 2oıo; Lojo, 2009):

Cansancio emocional: sensación de cansancio, pérdida de energía, agotamiento físico, fatiga, vacío. La persona se encuentra gastada, sin energía ni recursos emocionales y no puede dar más de sí.

Despersonalización: adopción de una actitud de distanciamiento emocional, irritabilidad, cinismo y endurecimiento emocional, con tendencia a considerar o percibir las personas como objetos insensibles. Aparece una notable pérdida de la capacidad de contacto interpersonal, insensibilidad y falta de empatía hacia los demás.

Baja realización personal: sentimiento de incapacidad, baja autoestima profesional, evaluación negativa de los resultados o de las realizaciones profesionales propias. El profesional siente que su competencia se reduce y se percibe cada vez más ineficaz e inútil. La autoestima disminuye progresivamente y como consecuencia se ve limitada, cada vez más, la capacidad para desarrollar con éxito el rol profesional. Este sentimiento de insatisfacción e infelicidad con el propio rendimiento laboral también se puede ir extendiendo a los ámbitos de la vida privada.

De este modo, según sus autoras, el burnout se inicia con agotamiento emocional, seguido de despersonalización y termina en baja realización personal. Importante ha sido esta definición, ya que, a partir de aquí, numerosos investigadores y organismos internacionales han intentado definir y determinar qué factores son los que desencadenan el burnout en un primer momento como, por ejemplo, los estudios de Golembiewski (1989), el cual concluye que para que aparezca el burnout primero debe incidir la despersonalización, luego la baja realización personal, terminando con el cansancio emocional. Por otra parte, Leiter y Maslach (200I) consideran que el cansancio emocional se convierte en despersonalización cuando se entra en conflicto con otro personas, perjudicando la realización personal. Gil-Monte (2005) manifestará 
que el síndrome del burnout viene determinado por una sensación de desbordamiento e impotencia relacionada con el entorno laboral. Comienza por una baja realización personal y cansancio emocional que conduce a la despersonalización como forma de afrontamiento del problema.

Una vez completado el marco conceptual y definidos los objetivos, se pueden formular las hipótesis de la investigación que se someten a contraste:

Hipótesis I: Los docentes que trabajan en los institutos públicos de educación secundaria obligatoria en las Illes Balears padecen burnout de manera significativa.

Hipótesis 2: Los docentes que trabajan en los institutos públicos de educación secundaria obligatoria en las Illes Balears sufren cansancio emocional de manera significativa.

Hipótesis 3: Los docentes que trabajan en los institutos públicos de educación secundaria obligatoria en las Illes Balears sufren despersonalización de manera significativa.

Hipótesis 4: Los docentes que trabajan en los institutos públicos de educación secundaria obligatoria en las Illes Balears padecen baja realización personal de manera significativa.

Hipótesis s: El síndrome del burnout se da por igual entre hombres y mujeres que trabajan en los institutos públicos de educación secundaria obligatoria de las Illes Balears.

\section{Desarrollo metodológico de la investigación}

Una vez desarrollada la aproximación conceptual del síndrome del burnout, es importante sentar las bases de un buen desarrollo metodológico para conseguir los objetivos propuestos; el diseño metodológico se desarrolla a partir de los objetivos e hipótesis: conocer las variables por las que se desarrolla el burnout, determinar en qué medida incide el síndrome del burnout en los profesores de centros públicos de secundaria de las Illes Balears y analizar si las medidas de prevención que se deben asumir para poder desarrollar un modelo de escuela los más saludable posible serán adecuadas.

Según Sánchez, «podemos ver claramente que a partir de la definición de Freudenberg en el año 1974, cada investigador ha desarrollado un nuevo modelo de evaluación con una media de un instrumento por año» (Sánchez, 2013), a partir de aquí, como instrumento, seleccionamos para nuestra investigación el Maslach Burnout Inventory (MBI), ya que es uno de los instrumentos de mayor calidad y más utilizados en las investigaciones sobre el burnout (Rubio, 2003). Este mismo autor manifiesta que no hay ningún instrumento que haya superado empírica o teóricamente al MBI. Anteriormente, Maslach y Jackson (1986) ya habían establecido que el Maslach Burnout Inventory (MBI) es la prueba psicológica más importante y aceptada por toda la comunidad científica. Por su parte, Buzzetti (2005) afirma que: «diversos autores reconocen que el MBI es el instrumento que más ha aportado a la conceptualización del síndrome (Cordes y Dougherty, I993; Gil-Monte, I999; Schaufeli, 2000²; Maslach,

2 Citado en Buzzetti Bravo (2005: 62). 
MALESTAR SOCIAL Y MALESTAR DOCENTE: UNA INVESTIGACIÓN SOBRE EL SÍNDROME DE... DAVID SÁNCHEZ LLULL, MARTÍ X. MARCH Y CERDÀ Y LLUÍS BALLESTER BRAGE

200I; Halbesleben, 2004)» y menciona que «solamente el MBI cuenta con muestras amplias y diversas en cuanto a características ocupacionales y de su lugar de origen, por lo que este instrumento ha sido aceptado como un estándar para medir el BO", siendo corroborado actualmente por el observatorio de riesgos psicosociales (Unión General de Trabajadores, 20I3). Por otra parte, esta postura queda reflejada también en la Revista Clínica Española de abril de 2009, la cual menciona en el artículo «Residentes, guardias y síndrome de burnout» las siguientes manifestaciones: «Para valorar el grado de burnout se utilizó el Maslach Burnout Inventory (мBI) considerado como el patrón oro de los cuestionarios para objetivar el burnout, el cual ha sido previamente validado en España» (Fonseca et al., 2009). A nivel europeo podemos citar el artículo «La validez factorial del Maslach Burnout Inventory-General Survey (MBI-GS) a través de los grupos profesionales y de las naciones», el cual apareció en la revista Journal of Occupational and Organizational Psychology. En ella se menciona que: «Poco después de su introducción en la década de 1980, el Maslach Burnout Inventory (MBI) se convirtió en el estándar de oro para evaluar el burnout» (Schütte, Toppinen, Kalima y Schaufeli, 20ro). Por último, a nivel internacional tenemos el proyecto RN4CAST (Registered Nurse Forecasting) de la Comisión Europea. Este es un proyecto de investigación financiado a través del VII Programa Marco de la Unión Europea que tiene como objetivo mejorar los modelos de previsión y planificación de los recursos humanos en enfermería, estudiando las condiciones laborales y las condiciones en que el personal de enfermería presta sus servicios. Participan once países europeos (Alemania, Bélgica, España, Finlandia, Grecia, Holanda, Irlanda, Polonia, Reino Unido, Suecia y Suiza) y cuatro países internacionales (Botswana, Estados Unidos, China y Sudáfrica). Según este consorcio: «El Maslach Burnout Inventory (MBI) es el patrón oro para medir los tres componentes del agotamiento debido al trabajo» (Comisión Europea, 20I2). Por otra parte, junto al MBI se pasará también un cuestionario sobre datos sociodemográficos de elaboración propia basadas en investigaciones que ponen de manifiesto la relación entre variables como el género, edad, experiencia docente y el burnout (Bresó, Salanova y Schaufeli, 2007; Larrote y Sáez, 2008).

El marco geográfico de nuestra investigación son cada una de las Illes Balears: Mallorca, Menorca, Eivissa y Formentera; cada una de estas islas cuenta con centros públicos de educación secundaria obligatoria.

Nuestro universo estaba formado por todos los profesores de la enseñanza secundaria pública obligatoria (ESO) que durante el año escolar 20Io/II hubiesen trabajado en los centros de la Comunidad Autónoma de las Illes Balears. De este modo, se analizó la estadística ofrecida por la Dirección General de Planificación y Centros, de la Consejería de Educación y Cultura del Gobierno de las Illes Balears sobre el número de profesores para el año 20Io, con el fin de determinar el tamaño mínimo que debe tener la muestra. Así, sabiendo que el universo estaba formado por 5.104 profesores, el número mínimo de profesores, con un nivel de confianza del 95,45\% y un error muestral del $5 \%$, era de 357 . Por tanto, se eligieron al azar nueve centros públicos de educación secundaria obligatoria, con un número total de 667 profesores. De los 667 cuestionarios repartidos, se recogieron 482 , es decir, un $72,2 \%$. De estos 482 cuestionarios, un 4,20\% se anuló por deficiencias a la hora de cumplimentarlos. Por lo tanto, quedó un total de 454 cuestionarios válidos, es decir, la muestra empírica final mejoraba la definición de la muestra teórica inicial, consiguiendo reducir el margen de 
error a un 3,89\%, en el supuesto de que los problemas derivados de la autocorrelación no fuesen significativos.

Para realizar el trabajo de campo, una vez seleccionados aleatoriamente ${ }^{3}$ los centros que se querían analizar y determinado el número de profesores, se solicitó permiso a las autoridades pertinentes y posteriormente se determinó quiénes eran las personas más adecuadas para ayudarnos a aplicar y recoger los cuestionarios MBI y el cuestionario de elaboración propia, los cuales debían ser profesores voluntarios que trabajasen en el mismo centro, a los cuales se les informó sobre la manera de proceder más adecuada en la investigación según el manual del мBi (Seisdedos, 1997).

Por último, mencionar que a la hora de aplicar los instrumentos, estos no se podían aplicar indiscriminadamente, sino que debían seguir unas pautas. Según el manual del мві de Maslach (Seisdedos, 1997), el Maslach Burnout Inventory podía ser aplicado individualmente o colectivamente. El instrumento no tiene un tiempo definido de aplicación aunque el tiempo medio que emplea un profesor en rellenarlo es de 3 a 5 minutos. Así, el MBI se presentó al claustro de profesores como una encuesta, mediante el personal antes seleccionado con el fin de crear un contexto adecuado y con el objetivo de recoger un cuestionario sincero y fiable. De esta manera, no debía ser aparente la intencionalidad de medir el burnout y, en consecuencia, el profesor colaborador no debía usar nunca esta terminología.

\section{Resultados de la investigación}

Como antecedentes a nuestra investigación, se constata que investigadores tan importantes como Manasero, Vázquez, Ferrer, Fornés y Fernández (2003) obtuvieron que un 40,03\% de profesorado de las Illes Balears padecían este síndrome. Por otra parte a nivel estatal, Arís (2009) nos hablaba también de cómo el burnout afectaba de forma moderada en su investigación y Rubio (2003) manifestaba que el $37 \%$ de los orientadores extremeños padecía burnout de forma significativa. Por otra parte, si comparamos nuestros resultados con la muestra de Seisdedos (1997), podemos afirmar que nuestra muestra es muy similar a los resultados de la muestra de docentes España N: 5I de la clasificación tripartita y estadística básica en muestras originales de los EE. UU. y España del manual del MBI (Seisdedos, 1997), excepto en realización personal, ya que los docentes de nuestras islas presentan menor realización personal.

Tabla i. Comparación de las medias de la Muestra Illes Balears N: 454 con la muestra de SeIsDedos (1997)

\begin{tabular}{|l|c|c|c|c|}
\hline Dimensiones del burnout & $\begin{array}{c}\text { Muestra total EE. } \\
\text { UU. N: } \mathrm{I} 067\end{array}$ & $\begin{array}{c}\text { Muestra total Es- } \\
\text { paña N: } \mathrm{I} 38\end{array}$ & $\begin{array}{c}\text { Muestra docentes } \\
\text { España N: } 5 \mathrm{I}\end{array}$ & $\begin{array}{c}\text { Estudio Sánchez } \\
\text { Llull N: } 454\end{array}$ \\
\hline Cansancio Emocional & 20,99 & 20,86 & 20,33 & 20.06 \\
\hline Despersonalización & 8,73 & 7,62 & 5,08 & 4,57 \\
\hline Realización Personal & 34,58 & $35,7 \mathrm{I}$ & 38,22 & 34,16 \\
\hline
\end{tabular}

3 Aleatorio simple. 
Si traducimos estos resultados a grados de burnout, coincidiendo con los percentiles 33 y 66, obtenemos que un $33,04 \%$ de los docentes presenta cansancio emocional de manera significativa, un $32,16 \%$ presenta despersonalización y un $32,38 \%$ padece de poca realización personal.

De esta manera, y en referencia a los últimos estudios sobre el burnout publicados en España de Arís (2009), Grau (2008) y Rubio (2003), podemos afirmar que nuestros resultados se acercan mucho a los resultados de estas investigaciones, excepto en realización personal, ya que los docentes de las islas presentan también menor realización personal. Por tanto, se corroboran las afirmaciones que hacen referencia a la incidencia del burnout de manera significativa.

TABLA 2. Cuadro comparativo de medias estudios recientes burnout en España

\begin{tabular}{|l|c|c|c|c|}
\hline Dimensiones del burnout & $\begin{array}{c}\text { Estudio Arís Redó } \\
(\mathrm{N}: 89)\end{array}$ & $\begin{array}{c}\text { Estudio Grau } \\
\text { Alberda (N: 316) }\end{array}$ & $\begin{array}{c}\text { Estudio Rubio } \\
\text { Jiménez (N: 65) }\end{array}$ & $\begin{array}{c}\text { Estudio Sánchez } \\
\text { Llull N: } 454\end{array}$ \\
\hline Cansancio Emocional & 20,66 & $\mathrm{I} 8,5 \mathrm{I}$ & 24,32 & 20,06 \\
\hline Despersonalización & 3,85 & 5,49 & 4,34 & 4,57 \\
\hline Realización Personal & $38, \mathrm{I3}$ & 35,99 & 36,65 & 34,16 \\
\hline
\end{tabular}

Por último y en referencia al género, vemos claramente que no existen diferencias significativas por razón de género en los profesores de enseñanza pública en secundaria de las Illes Balears.

TABLA 3. Comparación medias factores del burnout en hombres y mujeres

\begin{tabular}{|l|c|c|c|}
\hline Género & Cansancio emocional & Despersonalización & Realización personal \\
\hline Hombres & 20,27 & $5, \mathrm{II}$ & $3 \mathrm{I}, 95$ \\
\hline Mujeres & $\mathrm{I} 9,84$ & 4,28 & 34,63 \\
\hline Media & 20,06 & 4,69 & 33,29 \\
\hline Desviación estándar & 0,30 & 0,59 & $\mathrm{I}, 90$ \\
\hline Coeficiente de variación & $\mathrm{I}, 49 \%$ & $\mathrm{I} 2,58 \%$ & $5,71 \%$ \\
\hline
\end{tabular}

En conclusión y una vez estudiado el síndrome del burnout y analizada la prevalencia de este riesgo psicosocial entre el profesorado de los centros de enseñanza pública de secundaria de las Illes Balears, podemos afirmar que hemos detectado una incidencia significativa de este síndrome entre el profesorado de los centros públicos, corroborando también las afirmaciones de Riart y Martorell (20I0), Lojo (2009) y Longás (2010), en cuanto a la incidencia del burnout en los centros educativos, ya que se ha podido contrastar afirmativamente la hipótesis i: los docentes que trabajan en los institutos públicos de educación secundaria obligatoria en las Illes Balears padecen burnout de manera significativa. Más concretamente, la investigación muestra que el 33\% del profesorado sufre este riesgo psicosocial. En referencia a la prevalencia de los factores asociados al síndrome de burnout, se puede confirmar la hipótesis 2: los docentes que trabajan en los institutos públicos de educación secundaria obligatoria en las Illes Balears 
sufren cansancio emocional de manera significativa, ya que el 33,04\% del profesorado sufre cansancio emocional. En referencia a la hipótesis 3: los docentes que trabajan en los institutos públicos de educación secundaria obligatoria en las Illes Balears sufren despersonalización de manera significativa, se ha observado que un 32,16\% de la muestra presenta problemas de despersonalización. Por último, en referencia a la hipótesis 4: los docentes que trabajan en los institutos públicos de educación secundaria obligatoria en las Illes Balears padecen baja realización personal de manera significativa, obtenemos que un 33,I6\% de la muestra manifiesta tener bajos niveles de realización personal. Por otro lado, haciendo referencia a la diferencia de género, podemos decir que se puede confirmar la hipótesis 5: el síndrome del burnout se da por igual entre hombres y mujeres que trabajan en los institutos públicos de educación secundaria obligatoria en las Illes Balears, ya que las diferencias en cada una de las dimensiones del burnout no son significativas, como se comprueba en la siguiente tabla (4). TABLA 4. Comprobación presencia diferencial de los factores del burnout
en hombres y mujeres

\begin{tabular}{|l|c|c|c|}
\hline Estadísticos & Cansancio emocional & Despersonalización & Realización personal \\
\hline Chi-cuadrado de Pearson & $\mathrm{I}, 676$ & 0,369 & 2,047 \\
\hline Significación & 0,196 & 0,543 & 0,153 \\
\hline
\end{tabular}

\section{Conclusiones}

En base al estudio de las organizaciones más importantes a nivel internacional sobre la seguridad, salud y prevención de riesgos laborales, debemos decir que se ha podido constatar, de una manera u otra, la importancia del burnout como problemática psicosocial emergente que afecta a la salud de los trabajadores $y$, en consecuencia, a su rendimiento. Podemos afirmar que para la mayoría de organizaciones internacionales el burnout viene determinado por un gran agotamiento emocional, despersonalización y falta de realización personal. Por otra parte, no es extraño que dichas organizaciones manifiesten la necesidad de prestar más atención a los problemas psicosociales que afectan a la actividad laboral, ya que estos inciden en el rendimiento laboral, el cual está directamente relacionado con la eficacia de la organización. Cuanto peor es el modelo organizativo, peores son las medidas de prevención y esto puede incidir en la aparición del burnout y, en consecuencia, una disminución del rendimiento profesional. Es decir, existe una estrecha relación entre el modelo organizativo de la empresa/administración, prevención de riesgos laborales, seguridad y salud, burnout y rendimiento laboral. La relación sistémica que se establece entre deficiencias en la organización y burnout nos permite sospechar que el significativo nivel de dicha problemática en los centros públicos de secundaria de las Islas Baleares es un síntoma de diversas deficiencias de la organización que deberían ser exploradas e identificadas.

El problema de hoy en día es que se intenta en la mayoría de ocasiones actuar ante la problemática sin tratar la raíz del problema, es decir, una organización que no tiene en cuenta la prevención de riesgos laborales como manda la legislación internacional y comunitaria puede provocar la aparición de problemas psicosociales como el estrés crónico laboral, el cual evidentemente está relacionado con un pobre rendimiento 
laboral que, en el caso que nos ocupa, afectaría directamente a los profesores pero indirectamente a los alumnos con un alto índice de fracaso escolar.

En referencia al burnout, debemos decir que se ha encontrado niveles significativos de burnout en la muestra de profesores de los institutos de secundaria de las Illes Balears. Como resultados directos de la investigación tenemos que un porcentaje importante de profesores y profesoras son susceptibles de desarrollar la enfermedad o ya la sufren (33\% aproximadamente). En cuanto a las dimensiones que inciden en esta problemática, mencionar que el 33,04\% de la población padece cansancio emocional, un $32,16 \%$ de la muestra presenta problemas de despersonalización y un 33,16\% tiene poca realización personal. Es necesario recordar que para que se dé el síndrome del profesor quemado debe haber problemas de cansancio emocional, despersonalización y baja realización personal como los que se han encontrado.

Finalmente, para evitar el aumento de problemas psicosociales debidos a la falta de prevención y evaluación de los riesgos laborales, seguridad y salud y sus consecuencias como son unos resultados educativos negativos, proponemos que se trabaje para que la escuela sea un entorno saludable, es decir, una escuela crítica con ella misma, flexible, no burocrática, igualitaria (Longás, 20Io), que busca la armonía entre sus docentes a pesar de las problemáticas que puedan surgir, para lograr que sus alumnos se conviertan en personas libres que puedan cambiar un mundo cada vez más insostenible que evidentemente debe mejorar. Por otro lado y según el informe anual «contar con trabajadores saludables es clave para una recuperación económica sostenible». El doctor y director de la Agencia Europea para la Seguridad y la Salud en el Trabajo, Jukka Takala, manifiesta que: «Necesitamos garantizar no sólo que los empleos actuales sean seguras, saludables y productivas, también deberíamos esforzarnos por una vida laboral segura, saludable, productiva, sostenible, satisfactoria y llena de motivación» (EU-OSHA, 2OIO).

Todo ello debe plantearse no sólo en un proceso en el que las relaciones entre la escuela, la familia y la comunidad se desarrollen de forma coherente, sino en una mejora real de la figura del docente a nivel social, a nivel formativo, a nivel de imagen, a nivel de tratamiento en los medios de comunicación, etc. La dimensión socioeducativa de la problemática del malestar docente, del burnout, implica el desarrollo, también, de programas socioeducativos que posibiliten, junto con otras actuaciones -organizacionales, de salud laboral, de formación, de supervisión psicosocial, etc.- la mejora de este malestar de los docentes y de la mejora de la escuela en sus resultados, en su calidad y en su equidad.

\section{Bibliografía}

Agencia Europea para la Seguridad y Salud en el Trabajo (2002) Trabajemos contra el estrés. Revista de la Agencia Europea para la Seguridad y Salud en el Trabajo, 5, 6-8.

Agencia Europea para la Seguridad y Salud en el Trabajo (2005) El estrés. Recuperado el O4 de septiembre de 20I2, de http://osha.europa.eu/es/topics/stress/index_html.

Agencia Europea para la Seguridad y Salud en el Trabajo (20io) Informe anual eu-osha 20I0: contar con trabajadores saludables es clave para una recuperación económica sostenible.

Agencia Europea para la Seguridad y Salud en el Trabajo (20ir) What-is-burnout-andhow-to-prevent-it. Agencia Europea para la Seguridad y Salud en el Trabajo. Recuperado el 2I de julio de 2orI, de http://osha.europa.eu/en/faq/what-is-burnout-and-how-to-preventit/what-is-burnout. 

DAVID SÁNCHEZ LLULL, MARTÍ X. MARCH Y CERDÀ Y LLUÍS BALLESTER BRAGE

Aris Redó, N. (2009) El síndrome del burnout en los docentes. Facultad de Ciencias de la Educación. Universidad Internacional de Cataluña.

Asociación Nacional de Profesionales de la Enseñanza (20I3) Memoria estatal del defensor del profesor. Curso 20I2-13.

Boletín Oficial del Estado (1995) Ley 3i/1995, de 8 de noviembre, de Prevención de Riesgos Laborales. $B O E$, n. $^{\circ} 269$.

Bresó Esteve, E.; Salanova, M. y Schaufeli, W. (2007) NTP 732: Síndrome de estar quemado por el trabajo «Burnout» (III): Instrumento de medición. Instituto Nacional de Seguridad e Higiene en el Trabajo. Recuperado el 17 de febrero de 20I5, de http://www.insht.es/InshtWeb/Contenidos/Documentacion/FichasTecnicas/NTP/Ficheros/70Ia75o/ntp_732.pdf.

Buzzetti Bravo, M. (2005) Validación del Maslach Burnout Inventory (MBI), en dirigentes del Colegio de Profesores A.G. de Chile. Memoria para optar al título de Psicólogo. Facultad de Ciencias Sociales, Universidad de Chile.

COMisión Europea (2012) RN4CAST. Informe global hospitales españoles. Estrasburgo: Comisión Europea. Recuperado el 17 de febrero de 2015, de http://enfermeriacomunitaria.org/web/attachments/article/322/Informe\% $\%$ 20RN4CAST\% 2ohospitales\% 2oespan\% CC\% 83oles.pdf.

Esteve, J. M. (1987) El malestar docente. Barcelona: Laia.

FARber, B. (2000) Treatment Strategies for different types of teacher burnout. In session. Psychotherapy in practice, 56, 675-689.

http://dx.doi.org/I0.1002/(sici)I097-4679(200005)56:5<675::aid-jclp8>3.0.c0;2-d

Fonseca, M.; Sanclemente, G.; Hernández, C.; Visiedo, C.; Bragulat, E. y Miró, O. (2009) Residentes, guardias y síndrome de burnout. Revista Clínica Española, 5, 209-215.

Freudenberger, H. J. (1975) The staff burn-out syndrome in alternative institutions. Psycoterapy: Theory. Research and Practice, $\mathrm{I} 2,73-83$.

Gil-Monte, P. R. (2005) El sindrome de quemarse por el trabajo (Burnout). Una enfermedad laboral en la sociedad del bienestar. Madrid: Pirámide.

Gil-Monte, P. R. y Peiró, J. M. (1996) Un estudio sobre antecedentes significativos del «síndrome de quemarse por el trabajo» («burnout») en trabajadores de centros ocupacionales para discapacitados psíquicos. Revista de Psicología del Trabajo y de las Organizaciones, I2 (I), 67-80

Golembiewski, R. T. (1989) A Note on Leiter's Study, Highliting. Two Models of Burnout. Group E Organization Studies, I4 (I), 5-13. http://dx.doi.org/Io.II77/I05960II890I400I02

Govern DE LES Illes BALEARS (2OII) Instruccions per a l'organització i el funcionament dels centres docents públics d'educació secundària per al curs $201 I_{-I 2}$.

Grau Alberola, E. (2008) El sindrome de quemarse por el trabajo (Burnout) en profesionales de enfermeria: Un estudio longitudinal. Universitat de València: Servei de Publicacions.

Greene, G. (1960) Un caso acabado. Barcelona: Seix Barral.

Instituto de Evaluación (20Io) Sistema estatal de indicadores de la educación. Madrid: Ministerio de Educación.

Instituto Nacional de Seguridad e Higiene en el Trabajo (2006) nTP 704: Síndrome de estar quemado por el trabajo o «burnout» (I): definición y proceso de generación.

Larrote Reviriego, I. y SaÉz Carreras, J. (2009) ¿Cuál es el perfil epidemiológico de padecer burnout en profesores no universitarios de la región de Murcia? Revista Electrónica Interuniversitaria de Formación del Profesorado, I2 (I), 77-83.

Leiter, M. P. y Maslach, C. (200I) Burnout and Quality in a Sped-Up World. The Journal for Quality and Participation, 24, 48-5I.

Lojo Fritschi, F. (2009) El estrés en los docentes. Secretaría de Formación. Murcia: ANPeMurcia.

Longás Mayayo, J. (20IO) Una aproximació a l'escola com organització saludable. Anàlisi de la relació entre el context intern del docent $i$ la sindrome d'esgotament professional. Tesis doctoral. Barcelona: Universitat Ramon Llull. 
MALESTAR SOCIAL Y MALESTAR DOCENTE: UNA INVESTIGACIÓN SOBRE EL SÍNDROME DE... DAVID SÁNCHEZ LLULL, MARTÍ X. MARCH Y CERDÀ Y LLUÍS BALLESTER BRAGE

Manasero Mas, M. A.; Vázquez Alonso, A.; Ferrer Pérez, V. A.; Fornés Vives, J. y FernánDez Bennassar, M. C. (2003) Estrés y burnout en la enseñanza. Illes Balears: Edicions UiB.

Maslach, C. (1976) Burned out. Human Behavior, 5, 16-22.

Maslach, C. y Jackson, S. E. (198I) The measurement of experience burnout. Journal of Ocupational Behavior, 2, 99-113. http://dx.doi.org/IO.IO02/job.4030020205

Maslach, C. y Jackson, S. E. (1982) Burnout in health professions: a social psychological analysis. En G. S. SAnders y J. Suls (eds.) Social Psychology of Health and Illness. Hillsdale: LEA.

Maslach, C. y Jackson, S. E. (1986) Maslach Burnout Inventory (2. ${ }^{a}$ ed.). Palo Alto. California: Consulting Psychologists Press.

Ministerio de Educación. Gobierno de España (20IO) Abandono escolar prematuro. Sistema estatal de indicadores en la educación. Edición 2010.

Ministerio de Educación, Cultura y Deportes (20i2) Panorama de la Educación. Indicadores de la OCDE. Madrid.

Mourshed, M.; Patel, J. y Suder, K. (20I4) Getting Europe's youth into work. Nueva York, NY: McKinsey \& Company. Recuperado el I7 de febrero de 20I5, de http:// mckinseyonsociety.com/education-to-employment/europe-report/.

Novotec (2005) Informe de evaluación de factores psicosociales IES Emili Darder, I3.

OCDE (2009) Los docentes son importantes. Atraer, formar y conservar a los docentes eficientes. Recuperado el I2 de septiembre de 20I2, de http://www.waece.org/enciclopedia/2/Los docentes son importantes.pdf.

Organización Internacional del Trabajo (oIt) (20II) Programa y Presupuesto para 20I22013. Ginebra: Instituto Internacional de Estudios Laborales. Organización Internacional del Trabajo.

Ortega Esteban, J. (2002) La escuela como plataforma de integración. La educación social y la escuela ante los desafíos de una sociedad en transformación (violencia, racismo, globalización...). En V. NúÑEZ (coord.) La educación en tiempos de incertidumbre: las apuestas de la Pedagogía Social (pp. II3-I56). Barcelona: Gedisa.

Parcerisas Aran, A. (2008) Educación Social en y con la institución escolar. Pedagogía Social. Revista Interuniversitaria. (Barcelona, tercera época), 15-27.

Prieto Ursúa, M. y Bermejo Toro, L. (2005) Malestar docente y creencias de autoeficacia del profesor. Revista Española de Pedagogía, 63 (232), 493-510.

Red Mundial de Salud Ocupacional (200I) gOHNEt: Una Red Mundial de Salud Ocupacional para fortalecer la formación y la investigación en materia de salud en el trabajo, I.

Riart, J. y Martorell, A. (20IO) L'estrès laboral dels docents. Malalties que pot provocar $i$ propostes terapèutiques. Barcelona: ISEP.

Rubio Jiménez, J. C. (2003) Fuentes de estrés, sindrome de Burnout y actitudes disfuncionales en Orientadores de Instituto de Enseñanza Secundaria. Tesis doctoral. Universidad de Extremadura.

Sánchez Llull, D. (2013) Estrategias de afrontamiento del burnout en centros de secundaria. Educació i Cultura: revista mallorquina de Pedagogia, 24, 75-93.

Schutte, N.; Toppinen, S.; Kalimo, R. I. y Schaufeli, W. (20Io) The factorial validity of the Maslach Burnout Inventory-General Survey (MBI-Gs) across occupational groups and nations. Journal of Occupational and Organizational Psychology, 73, 53-66. http://dx.doi.org/Io.I348/o96317900I66877

Seisdedos, N. (1997) mbi. Inventario «Burnout» de Maslach. Publicaciones de Psicología Aplicada, 22I. Madrid: Tea Ediciones.

Tortella-Feliu, M. (200I) L'estrès ocupacional en els mestres d'escola. Palma: Universitat de les Illes Balears. Servei de Publicacions.

Unión General de Trabajadores (2013) Observatorio de riesgos psicosociales. Ficha de prevención, 42. Recuperado el I7 de febrero de 2015, de http://www.ugt.es/ saludlaboral/observatorio/fichas/FichasObservatorio \% 2042.pdf. 